\title{
MODEL ORDER SELECTION FOR MULTIDIMENSIONAL INNOVATIONS BASED DETECTION IN AIRBORNE RADAR $^{12}$
}

\author{
Julio Castro James P. LeBlanc \\ julcastr@nmsu.edu leblanc@nmsu.edu \\ Klipsch School of ECE \\ New Mexico State University \\ Las Cruces, NM USA
}

\begin{abstract}
This paper investigates the model order selection problem for use with the multidimensional autoregressive (MAR) process in airborne radar detection processing which uses an Innovations Based Detection Algorithm (IBDA). Results indicate that a low order model should be used to accurately portray the return signal spectrum. Specifically, this paper investigates the use of the Akaike Information Criterion for model order selection. Examples are included for physically modeled data sets as well as actual radar data sets.
\end{abstract}

\section{INTRODUCTION}

Parametric modeling using autoregressive processes has been used extensively in the literature for various applications in the univariate case as well as in the multivariate case (Kay, 1988) (Jones, 1974) (Nuttall, 1976). However, literature discussing the application of multidimensional autoregressive (MAR) processes is limited for the airborne RADAR target detection application (Michels, 1991). Noticeably absent is guidance and recommendations for model-order selection for this application. In this research order selection and applicability of MAR processes to airborne RADAR surveillance target detection is investigated.

The use of the MAR structure to model the processes associated with airborne RADAR surveillance scenario lies in the fact that a target return maybe considered as an impulse in its frequency domain. Since data that contains spectral peaks may be well-modeled by AR processes (Kay, 1988), then it is not difficult to assert that a MAR process with a specified order could be used for model-based target detection. Initial results of such observations are presented herein, as well as in (LeBlanc, 1996) and (Michels, 1998); such experiments show that the order demanded for such an application is relatively low. This is a critical point for airborne surveillance RADAR applications since detection algorithms must be feasible to implement in real-time with limited computational resources.

An important aspect of MAR processes is the determination of the model order. For the most part, the selection of the model order dictates how well model will follow the true spectrum. A model order that is too high will cause spurious peaks in the frequency response as well as a high parameter variance. A too low model order will cause unwanted smoothing of the exhibiting spectrum (Kay, 1988). Both cases (too high or too low) will adversely affect detection performance. Several researchers have introduced criteria for model order use for specific scenarios (Akaike, 1971). The use of the Akaike Information Criteria (AIC), is explored in this research for the airborne RADAR signal return which can be regarded as a complex vector time-series signal.

In section 2, the airborne surveillance RADAR detection problem and the multidimensional AR theory is presented more detail. The $A I C$ for the multivariate case is discussed in section 3. Numerical results using synthetic and actual radar data are presented in section 5 .

\footnotetext{
${ }^{1}$ Supported by the United States Air Force, contract number: F49620-93-C-0063A

${ }^{2}$ Presented at the IEEE 1998 National Radar Conference, Dallas, Tx, 12-13 May 1998
} 


\section{RADAR TARGET DETECTION/MAR PROCESS}

In general, the airborne surveillance radar target detection consists in determining whether a target is present or not in a large number of range cells. Additionally, if a target is present, estimating its speed and range is desired. This problem has been studied extensively since the conception of RADAR technology; however, new methods continue to appear in the literature. A driving force behind continued research in this problem is that there is always the need to detect the target faster, cheaper (economically and/or computationally) and more accurately as well as robustly.

A received RADAR return at the $J$ element array for range cell $m$ can be represented in matrix form as,

$$
X(m)=\left[\begin{array}{cccc}
x_{1}(1) & x_{1}(2) & \cdots & x_{1}(N) \\
\vdots & \vdots & \ddots & \vdots \\
x_{J}(1) & x_{J}(2) & \cdots & x_{J}(N)
\end{array}\right]
$$

where $N$ is the number of pulses in a coherent processing interval (CPI). In general, each signal component, $x_{i}(n)$, at the $i$ th array element and at time $n$ is equal to

$$
x_{i}(n)=s(n)+j(n)+c(n)+w(n)
$$

where $s(n)$ is the target return reflection, $j(n)$ is a possible jammer, $c(n)$ is clutter reflection, and $w(n)$ is a white noise component. The covariance matrix $R_{x x}(k)$ of signal $x$ is the $J \times J$ matrix

$$
R_{x x}(k)=\left[\begin{array}{cccc}
r_{11}(k) & r_{12}(k) & \cdots & r_{1 J}(k) \\
\vdots & \vdots & \ddots & \vdots \\
r_{J 1}(k) & r_{J 2}(k) & \cdots & r_{J J}(k)
\end{array}\right]
$$

where each element $r_{i j}(k)=E\left\{x_{i}(n)^{*} x_{j}(n+k)\right\}$.

A MAR process can be described by the following equation

$$
X(n)=-\sum_{k=1}^{p} A(k) X(n-k)+U(n),
$$

where $A(k) \in \mathbb{C}^{J \times J}$, are the complex feedback parameter matrices, $X(n) \in \mathbb{C}^{J}$ is the data vector, and $U(n)$ is a multichannel white input process whose autocorrelation function satisfies $R_{u u}(k)=\Sigma \delta(k)$, where $\Sigma$ is the variance of the process. Notice that this type of modeling can also be viewed as an innovation process. The idea behind IBDA is that the prediction filter coefficients $A(k)$ would contain information about the received signal (clutter, jamming, signal), and hence a detection test could be based upon the achieved parameterization of the $A(k) \mathrm{s}$. A solution for finding the set of MAR parameters $\{A(k), 1 \leq k \leq p\}$ is by solving the multidimensional Yule-Walker equations. The estimated driving covariance matrix is given as follows

$$
\hat{\Sigma}=R(0)+\sum_{k=1}^{p} R^{H}(k) A^{T}(k)
$$

where $\hat{X}(n)$ is the forward prediction define as $\hat{X}(n)=-\sum_{k=1}^{p} A(k) X(n-k)$. Notice that the prediction error power, here called PEP, is equal to the trace of $\hat{\Sigma}$ and the off diagonals elements correspond to the (spatial correlation) cross-correlation of the driving noise.

\section{MULTIDIMENSIONAL AR MODEL ORDER}

The $A I C$ for real MAR processes was first introduced by Akaike in (Akaike, 1971) and is one of the model order indicators most cited. The $A I C$ criterion for real MAR processes (Kay, 1988) is given by

$$
A I C(p)=N \ln (\|\hat{\Sigma}\|)+2 J^{2} p
$$


where $p$ is the model order, $\hat{\Sigma}$ is the $p$ th order estimate of the prediction error covariance matrix, $J$ is the number of channels, and $\|\cdot\|$ indicates the determinant operation. Notice that since (6) was developed for real data, the direct application to the multichannel airborne radar problem (with complex signals) is not readily apparent. Nuttall (Nuttall, 1976) presented an upper bound on the model order usable with the $A I C$. This upper bound is given by

$$
\max \left(p_{A I C}\right) \leq \frac{3 \sqrt{N}}{J} .
$$

For the present work, (7) can also be interpreted as a relation between the prediction error power and the model order for a specific data length. The prediction error power simulations for independent radar return data sets presented in the next section verify this relation and show that $A I C$ results for $p$ above this upper bound should be used cautiously. For the present airborne radar case, a typical return data length is short and complex, thus limiting the usefulness of the $A I C$. The experimental simulations with independent realizations of return data also show that for large model order there is a reduction in prediction error power but at the expense of parameter variance. This leads to large prediction error power and thus could serve as an indicator for model order selection.

\section{COMPUTER SIMULATIONS}

In this section computer simulations are presented for the prediction error power, model order selection, and prediction error power for independent data realizations of modeled data as well as actual radar data from the Multi-Channel Airborne Radar Measurement (MCARM) project. The simulated return data were generated by routines developed by Scientific Studies Corporation (Roman, 1996). The parameters used in the simulation are given in table 1.

Table 1: SIMULATION PARAMETERS

\begin{tabular}{|r|l|}
\hline$J=14$ & Number of array elements \\
$N=64,128,200,550$ & Number of pulses in one CPI \\
$\phi_{0}=30$ & Array main beam azimuth angle \\
$f_{P R F}=300$ & Pulse repetition frequency $(\mathrm{Hz})$ \\
$f_{C}=450$ & Transmit frequency $(\mathrm{MHz})$ \\
$U N I F O R M$ & Array pattern \\
$H_{p}=9$ & Platform altitude $(\mathrm{km})$ \\
$V_{p}=25$ & Platform velocity $(\mathrm{m} / \mathrm{s})$ \\
$r_{c}=130$ & Range to ground clutter ring $(\mathrm{km})$ \\
$\gamma=20$ & Aircraft platform crab angle \\
$N_{c}=361$ & Number of clutter patches \\
$v a r n=1$ & Noise power in each channel \\
$S N R=3$ & Target signal-to-noise ratio $(\mathrm{dB})$ \\
$f_{s}$ & Spatial Frequency $\{-0.25\}$ \\
$f_{t}$ & Temporal Frequency $\{0.25\}$ \\
$N r=10$ & Number of realizations \\
\hline
\end{tabular}

\subsection{AIC}

Figure 1 show $A I C$ plots for simulated data of lengths $64,128,200$, and 550. In general, the proper model order is usually that which minimizes the $A I C$; however, notice that these figures do not show the expected concave behavior for the $A I C$ curves. These $A I C$ curves are better explained in conjunction with the Nuttall upper bound. Notice that for the 64 points data set the minimum $A I C$ is 8 , while on the contrary the Nuttall upper bound for this data set dictates that the order do not exceed 1.7. This is indicative that the AIC should be used cautiously for such short data sets. 


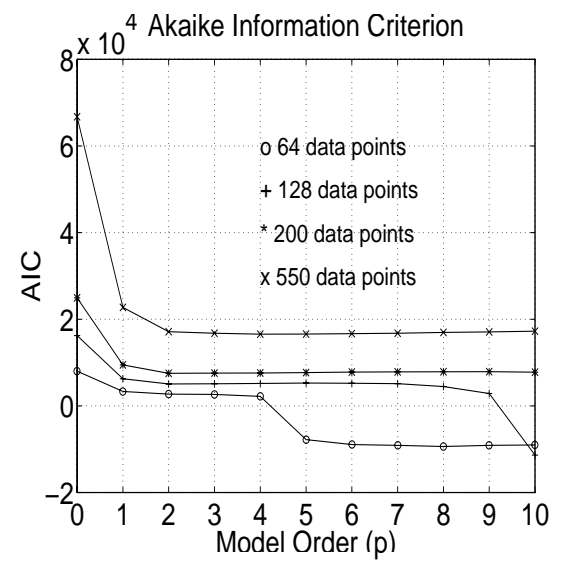

Figure 1: AIC for 64, 128, 200, and 550 Data Points

\subsection{PEP FOR INDEPENDENT REALIZATIONS}

Due to the limitations of the $A I C$, an alternative means of deciding model order follows. Model order selection information can be extracted from analysis of the Nuttall upper bound and the prediction error power (PEP) of independent data realizations. Figures $2,3,4$, and 5 show the prediction error power for simulated airborne return data of lengths $64,128,200$, and 550. One salient feature of these prediction error power plots are that the difference between the PEP of order 1 and those of higher order are within $2.5 \mathrm{~dB}$. This means that any increase in the model order does not contribute significantly to any additional prediction error reduction.

These plots also show that the average PEP for a set of independent data realizations varies dramatically after the Nuttall upper bound. This indicates that the parameters for one data set applied to any independent data set have a relatively small variance for model orders less than the Nuttall limit. This shows that for short data sets, prediction error power analysis in conjunction with the Nuttall upper bound provide a crude indicator for the model order to use.

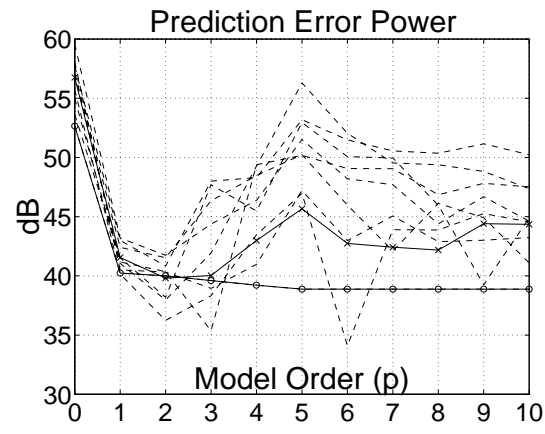

Figure 2: 64 Data Points

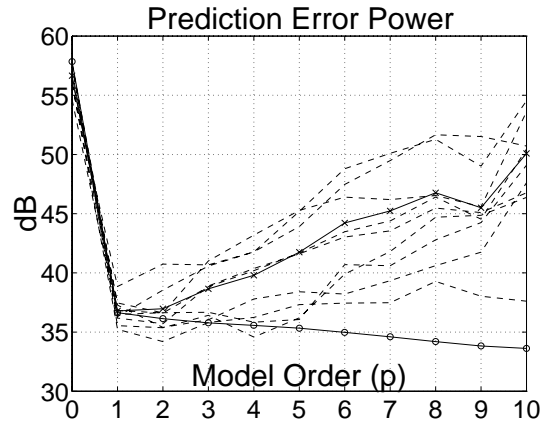

Figure 3: 128 Data Points

\subsection{MCARM DATA}

This section presents simulations using MCARM data. A typical AIC curve for this type of data is shown in Figure 6. Notice that this curve does not show the expected concave behavior since the data relatively short time support (128 time samples).

Simulation results show that the prediction error power decreases with respect to the model order. In some cases the prediction error power decreases from about $2 \mathrm{~dB}$ to about 10dB. This might indicate that using a high model order will lead to a more accurate prediction of the data. However, the upper limit for the model order should not exceed 1.7 for the time support considered in these simulations. Moreover, if 


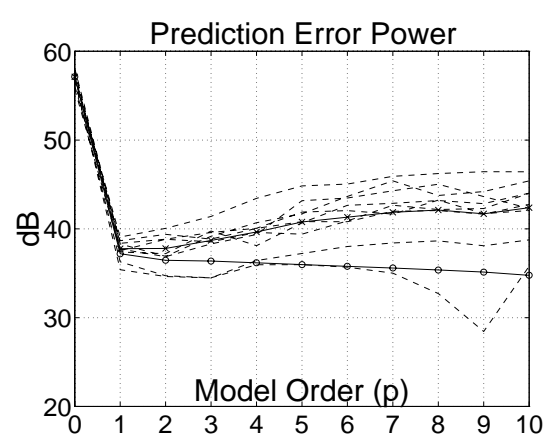

Figure 4: 200 Data Points

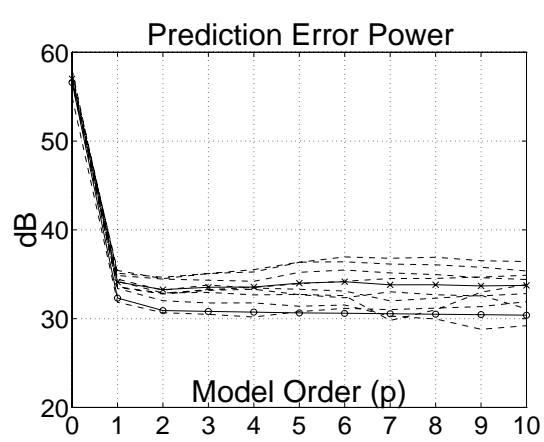

Figure 5: 550 Data Points

higher model orders are considered then the MAR model leads to undesire spurious peaks in the spectrum.

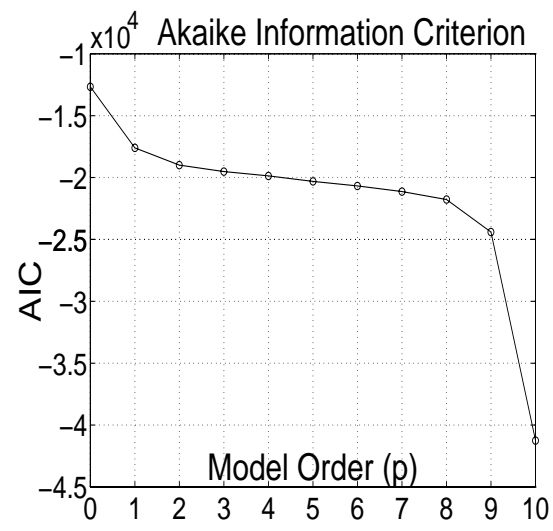

Figure 6: Typical AIC for MCARM Data

\subsection{MCARM PEP FOR INDEPENDENT REALIZATIONS}

Figures 7 through 10 show the prediction error power of independent realizations, where the independent data realizations are related to different range cells for a particular data acquisition. The data under study is that of range cell 200 for the different acquisitions and the average prediction error power was taken over 9 independent realizations. In particular, the independent realizations are those of range cells 120, 140, 160, 180,220,240,260,280, and 300. Notice that all the plots show similar behavior as that of the simulated data. That is, the average PEP for a set of independent realizations varies dramatically after a relatively small model order, except for acquisition \#520 which do not show this general behavior. This unexpected behavior might be attributed to the terrain from which this data was obtained.

\section{CONCLUSION}

The simulations results for the simulated and actual data sets indicate that the $A I C$ model order selection criterion must be used cautiously for the time support considered in the airborne scenario. However, the general behavior of the average prediction error power for independent realizations of both the simulated and actual data show that a low model order MAR model can be used. However, more research is needed to verify the theoretical background of this heuristic observation. In addition, an extension of the $A I C$ to the multidimensional complex scenario and investigation of model order selection using other model order criteria for return airborne data is needed. 


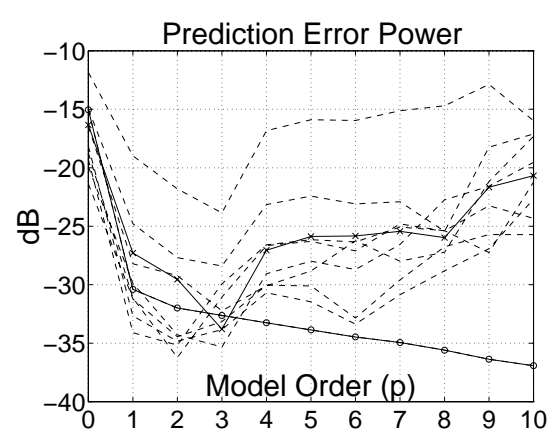

Figure 7: Acquisition \#465

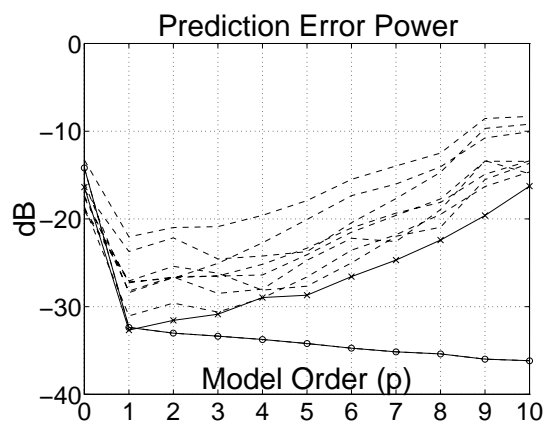

Figure 9: Acquisition \#575

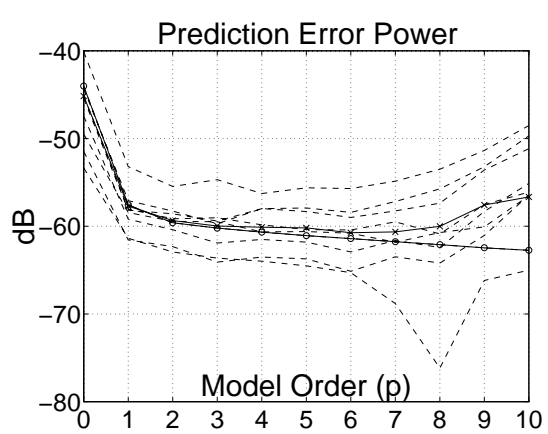

Figure 8: Acquisition \#520

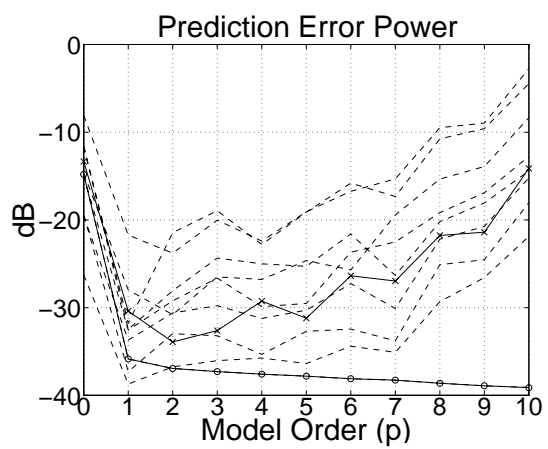

Figure 10: Acquisition \#628

\section{References}

[1] H. Akaike, "Autoregressive Model Fitting for Control," Ann. Inst. Statist. Math., Vol. 23, pp. 163-180, 1971.

[2] Richard H. Jones, "Identification and Autoregressive Spectrum Estimation," IEEE Trans. Autom. Control, Vol. AC-19, No. 6, pp. 894-897, Dec 1974.

[3] S. M. Kay, Modern Spectral Estimation: Theory \& Application, New Jersey: Prentice-Hall, Inc., 1988.

[4] J. P. LeBlanc, "Multichannel Autoregressive Modeling and Spectral Estimation Methods for Airborne Radar Environment," AFOSR, Final Report, pp. 16-1-16-19, Aug. 1996.

[5] J. H. Michels, "Multichannel Detection Using The Discrete-Time Model-Based Innovations Approach," Ph.D Dissertation, Syracuse University, New York, May 1991.

[6] J. Michels, T. Tsao, B. Himed, and M. Rangaswamy, "Space-Time Adaptive Processing (STAP) in Airborne Radar Applications," International Conference Signal Processing and Communications, Canary Islands, Spain, February 11-14, 1998.

[7] A. H. Nuttall, "Multivariate Linear Predictive Spectral Analysis Employing Weighted Forward and Backward Averaging: A Generalization of Burg's Algorithm," Naval Underwater System Center, New London, CT. Tech. Rep. 5501, Oct. 13, 1976.

[8] J. R. Roman, D. W. Davis, "Multichannel System Identification using Output Data Techniques Vol II Final Report No. SSC-TR-96-02," Scientific Studies Corp., Palm Beach Gardens, FL, October, 1996. 